\title{
Review of: "Mutations of CX46/CX50 and Cataract Development"
}

\author{
Anaclet Ngezahayo ${ }^{1}$ \\ 1 Universität Hannover
}

Potential competing interests: The author(s) declared that no potential competing interests exist.

The lens is an avascular organ constituted of layers of fiber cells. The most central fiber layer is formed very early in the embryological development and new layers are continuously added as the lens develops, through the fetal and the juvenile development and even in the adulthood. As a large avascular organ, the nutrients and liquid homeostasis of the lens is maintained by a complex lens microcirculation system in which, connexin channels formed by Cx46 and Cx50 take a crucial role. Formation of cell-to-cell gap junction channels and eventually connexin hemichannels is the most recognized role of connexins. In the lens bulk, Cx46 and Cx50 form the gap junction channels between the lens fibers and Cx46-hemichannels has been shown to participate to the $\mathrm{Na}^{+}$- leak conductance of the lens fibers (Ebihara et al 2014). The present review by Shi et al (2022) clearly and concisely summarizes recent findings concerning Cx46/Cx50 mutations that are linked to lens cataracts and failure in lens development. It is obvious that our understanding of how the mutations are linked to dysfunction of connexin channels and thus to cataract or to failure in lens development are more descriptive than mechanistic. This is due to a lacking understanding of degree of gap junction coupling and the activity of Cx-hemichannels in the lens fibers. Secondly, little is known about the selectivity of the Cx-channels (gap junction channels and hemichannels) for specific nutrients and substances relevant for both the development and the physiological homeostasis of the lens. This aspect could be a very interesting research topic. Further truncation of the connexins molecules during lens aging has been shown as a special posttranslational modified of the connexins in the lens. As clearly described in the review (Shi et al 2022), the physiological significance of the truncation is still matter of speculation. It was proposed that the truncation of the connexins conferred to the channels a reduced sensitivity to the acidic pH or to increased oxidative stress of the core of the lens (Salvi et al 2016, Wang and Schey 2009; Yin et al 2001). It is also possible that the truncation allows the remodeling the cellto-cell adhesion in old lens layers, allowing thereby a new positioning of the gap junction channels as the lens fiber get older (Jacobs et al 2004). We have also shown that the truncation of the C- terminus of Cx46 to a certain extend did not affect the capacity of the protein to form gap junction channels but reduced the formation of annular junctions suggesting that the truncation could affect the removal of the gap junction channels from the cell membrane (Schlingmann et al 2013). The idea could cope very well with the fact that old lens fiber has neither nuclei nor ER nor Golgi apparatus which means that the capacity to renew their membrane proteins is almost nil. Old fibers need mechanism to avoid the removal of protein out of 
the membrane. The truncation of the C-terminus could be one of the mechanisms.

The present review resumes very well the current knowledge about Cx46 and Cx50 mutations with respect to alteration of the lens development and lens cataract. The review stress also the need of further combined biophysical, genetic and functional research to make our understanding of how $\mathrm{Cx}$ mutations affect the development of the lens and the cataract.

\section{References}

Ebihara L, Korzyukov Y, Kothari S, Tong JJ (2014). Cx46 hemichannels contribute to the sodium leak conductance in lens fiber cells. Am J Physiol Cell Physiol 306:C506-C513 doi: 10.1152/ajpcell.00353.2013 Jacobs MD, Soeller C, Sisley AM, Cannell MB, Donaldson PJ (2004). Gap junction processing and redistribution revealed by quantitative optical measurements of connexin46 epitopes in the lens. Invest Ophthalmol Vis Sci 45:191-199.doi: 10.1167/iovs.03-0148

Schlingmann B, Schadzek P, Hemmerling F, Schaarschmidt F, Heisterkamp A, Ngezahayo A (2013). The role of the C-terminus in functional expression and internalization of rat connexin46 (rCx46). J Bioenerg Biomembr 45:59-70. doi: 10.1007/s10863-012-9480-x

Shi Y, Li X, Yang J (2022) Mutations of Cx46/Cx50 and cataract development. Front Mol Biosci 9:842399. doi: 10.3389/fmolb.2022.842399

Slavi N, Wang Z, Harvey L, Schey KL, Srinivas M. (2016) identification and functional assessment of agedependent truncation to Cx46 and Cx50 in the human lens Invest. Ophthalmol Vis Sci 57:5714-5722. doi: 10.1167/iovs.16-19698

Wang Z, Schey KL (2009). Phosphorylation and truncation sites of bovine lens connexin 46 and connexin 50. Exp Eye Res 89:898-904. doi: 10.1016/j.exer.2009.07.015

Yin X, Gu S, Jiang (2001). The development-associated cleavage of lens connexin 45.6 by caspase-3-like protease is regulated by casein kinase II-mediated phosphorylation. J Biol Chem 276:34567-34572. doi: 10.1074/jbc.M106073200 